Hiroyuki Yano, MD

Division of Rheumatology,

Department of Internal Medicine,

Okinawa Chubu Hospital,

Okinawa, Japan
Mitsuyo Kinjo, MD, MPH

Division of Rheumatology,

Department of Internal Medicine,

Okinawa Chubu Hospital,

Okinawa, Japan

\title{
Cutaneous digital papules
}

\begin{abstract}
$A^{5+12}$ 5I-YEAR-OLD MAN presented with creamcolored papules on his fingers. Ten years before, he began to have intermittent attacks of foot pain 2 or 3 times a year. Then, 3 years before this presentation, he developed nodules on both elbows and painless papules on the finger pads of both hands. He received a diagnosis of gout and stage $3 \mathrm{~b}$ chronic kidney disease, and urate-lowering therapy was initiated at a local clinic. He had had no recurrence of gouty attacks since initiation of this therapy. However, the therapy was not titrated, and he did not reach the serum urate goal. His renal function gradually declined, and he was referred to our hospital.

His medications at the time of presentation included febuxostat $10 \mathrm{mg}$ daily and benzbromarone (a uricosuric not available in the United States) $25 \mathrm{mg}$ daily. He was an executive of a construction company. He said he drank approximately $500 \mathrm{~mL}$ of Japanese sake twice a week for the past 30 years. He had no family history of gout.

His vital signs were stable. Physical examination revealed large nodules $(7 \mathrm{~cm})$ on both elbows, with similar but smaller lesions on the right lateral malleolus $(2 \mathrm{~cm})$ and the right second metatarsophalangeal joint $(1 \mathrm{~cm})$, and multiple nontender papules on all fingers, especially the pads (Figure 1).

Laboratory testing showed a serum uric acid level of $9.9 \mathrm{mg} / \mathrm{dL}$ (reference range 3.7$7.8 \mathrm{mg} / \mathrm{dL}$ ), blood urea nitrogen $39 \mathrm{mg} / \mathrm{dL}$ (8$20 \mathrm{mg} / \mathrm{dL})$, creatinine $2.80 \mathrm{mg} / \mathrm{dL}$ (0.65-1.07 $\mathrm{mg} / \mathrm{dL}$ ), and estimated glomerular filtration rate $20.5 \mathrm{~mL} / \mathrm{min} / 1.73 \mathrm{~m}^{2}$.

Radiography of the elbows revealed erosions of the distal humerus and increased nodular soft-tissue density. Aspiration of the nodule on the left elbow revealed needle-shaped
\end{abstract}

doi:10.3949/ccjm.88a.20063

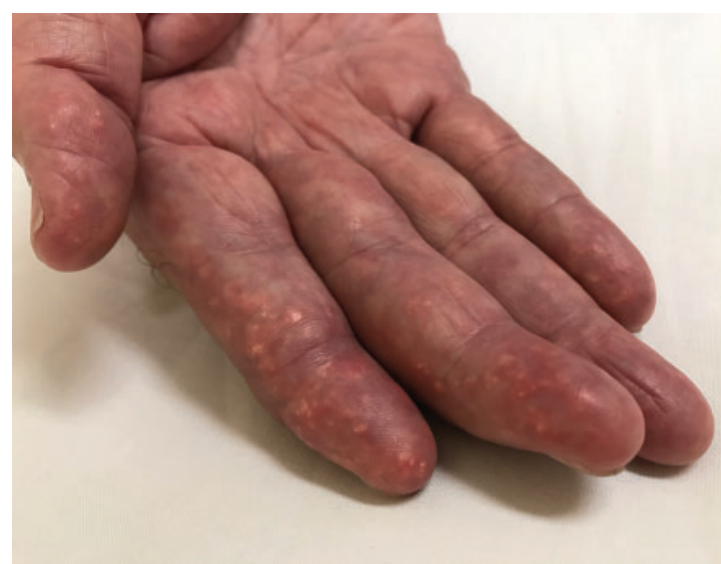

Figure 1. Multiple nontender papules were noted on all fingers.

crystals exhibiting characteristic negative elongation (birefringence) on compensated polarized light microscopy (Figure 2), leading to a diagnosis of tophaceous gout. Febuxostat was increased to $30 \mathrm{mg}$ daily. One year later, he remained free from gouty attacks, with regression of tophi and a uric acid level of 6.6 $\mathrm{mg} / \mathrm{dL}$.

\section{TOPHACEOUS GOUT: KEY FEATURES}

Gout, which affects $3.9 \%$ of US adults, is a metabolic disease that causes acute inflammatory arthritis, gouty tophi, renal impairment, and kidney stones (calcium oxalate and uric acid nephrolithiasis). ${ }^{1}$ Comorbidities such as hypertension, chronic kidney disease, obesity, diabetes mellitus, myocardial infarction, and stroke are common in patients with gout. ${ }^{2}$

Gout has 4 clinical stages; asymptomatic hyperuricemia, acute gouty arthritis, intercritical gout, and advanced tophaceous gout. ${ }^{3}$ Gouty tophi typically develop in the fourth stage and present more than 10 years after the first episode of acute gouty arthritis. ${ }^{2}$ Tophi
Tophi in the finger pads is a compelling indication for aggressive urate-lowering therapy 


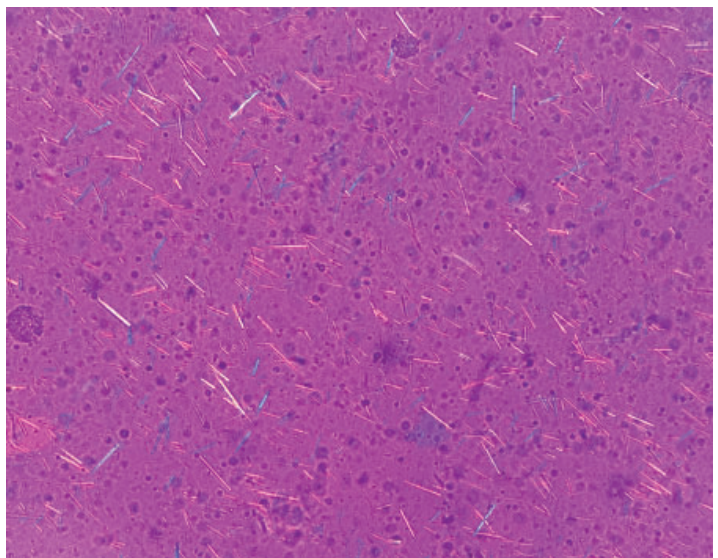

Figure 2. Aspiration of the nodule on the left elbow revealed needle-shaped crystals exhibiting characteristic negative elongation (birefringence) on compensated polarized light microscopy (magnification $\times 400$ ).

represent a granulomatous inflammatory response triggered by exposure to monosodium urate crystals. ${ }^{3}$

\section{FINGER PAD TOPHI NOT SO UNCOMMON}

Tophaceous gout typically presents at articular or periarticular sites, but uric acid crystals may also deposit in extra-articular sites. ${ }^{4}$ Finger pad tophi are superficial intradermal collections of monosodium urate. Although thought to be a rare manifestation of advanced gout, they may be overlooked clinically. In a small patient series, ${ }^{5}$ finger pad tophaceous deposits were observed in $11(30.5 \%)$ of 36 patients with tophaceous gout.

Risk factors for extra-articular tophi are renal insufficiency, hypertension, long-term use of furosemide, and long duration of disease. ${ }^{3}$ The differential diagnosis of papules on finger pulp includes rheumatoid nodule, calcinosis cutis, pyogenic pustules, and fibromatosis.,

Treatment of gout has four dimensions, ie, urate-lowering therapy (in patients who qualify), flare prophylaxis, flare management, and management of gout-associated comorbidities. Tophi alone are an indication for urate-lowering therapy such as allopurinol, based on the recommendation of the American College of Rheumatology.

\section{TAKE-HOME POINTS}

Development of tophi in the finger pads constitutes a compelling indication for aggressive urate-lowering therapy, which may prevent severe degenerative arthritis, secondary infections, and gout-associated comorbidities. We should perform a full physical examination on all gout patients, as tophi can develop in extraarticular locations including the finger pads, helix of the ear, and olecranon bursa.

\section{DISCLOSURES}

The authors report no relevant financial relationships which, in the context of their contributions, could be perceived as a potential conflict of interest.

\section{REFERENCES}

1. Chen-Xu M, Yokose C, Rai SK, Pillinger MH, Choi HK. Contemporary prevalence of gout and hyperuricemia in the United States and decadal trends: the National Health and Nutrition Examination Survey, 2007-2016. Arthritis Rheumatol 2019; 71(6):991-999. doi:10.1002/art.40807

2. Dalbeth N, Merriman TR, Stamp LK. Gout. Lancet 2016; 388(10055):2039-2052. doi:10.1016/S0140-6736(16)00346-9

3. Chhana A, Dalbeth N. The gouty tophus: a review. Curr Rheumatol Rep 2015; 17(3):19. doi:10.1007/s11926-014-0492-x

4. Çerman AA, Altunay IK, Salman KE, Tunçel D. Finger pad tophi in gout: a rare presentation. An Bras Dermatol 2016; 91(6):855-856. doi:10.1590/abd1806-4841.20164466

5. Holland NW, Jost D, Beutler A, Schumacher HR, Agudelo CA. Finger pad tophi in gout. J Rheumatol 1996; 23(4):690-692. pmid:8730128

6. Braun-Falco M, Hofmann SC. Tophaceous gout in the finger pads. Clin Exp Dermatol 2010; 35(3):e22-e23. doi:10.1111/j.1365-2230.2009.03331.x

7. FitzGerald JD, Dalbeth N, Mikuls T, et al. 2020 American College of Rheumatology guideline for the management of gout. Arthritis Care Res (Hoboken) 2020; 72(6):744-760. doi:10.1002/acr.24180

Address: Mitsuyo Kinjo, MD, MPH, Division of Rheumatology, Department of Internal Medicine, Okinawa Chubu Hospital, 281 Miyazato, Uruma City, Okinawa, 904-2293, Japan; kinjomitsuyo@gmail.com 